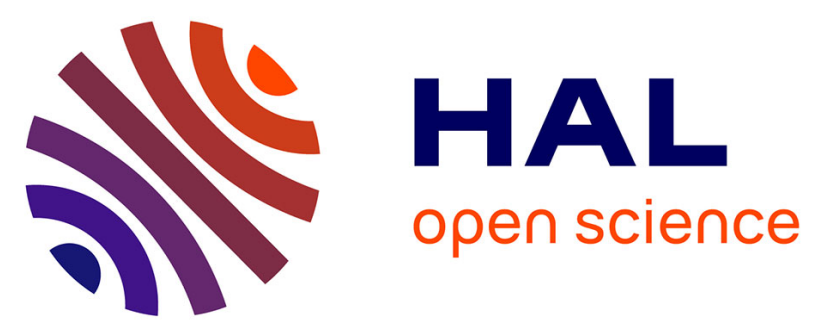

\title{
Présence en réunion de concertation pluridisciplinaire dans les cancers des voies aérodigestives supérieures : vécu et satisfaction du patient
}

D. Chaillou, G. Mortuaire, V. Deken-Delannoy, B. Rysman, D. Chevalier, F. Mouawad

\section{To cite this version:}

D. Chaillou, G. Mortuaire, V. Deken-Delannoy, B. Rysman, D. Chevalier, et al.. Présence en réunion de concertation pluridisciplinaire dans les cancers des voies aérodigestives supérieures : vécu et satisfaction du patient. Annales françaises d'Oto-rhino-laryngologie et de Pathologie Cervico-faciale, 2019, 136, pp.76 - 83. 10.1016/j.aforl.2018.03.007 . hal-03486515

\section{HAL Id: hal-03486515 \\ https://hal.science/hal-03486515}

Submitted on 20 Dec 2021

HAL is a multi-disciplinary open access archive for the deposit and dissemination of scientific research documents, whether they are published or not. The documents may come from teaching and research institutions in France or abroad, or from public or private research centers.
L'archive ouverte pluridisciplinaire $\mathbf{H A L}$, est destinée au dépôt et à la diffusion de documents scientifiques de niveau recherche, publiés ou non, émanant des établissements d'enseignement et de recherche français ou étrangers, des laboratoires publics ou privés.

\section{다)(1) $(5$}

Distributed under a Creative Commons Attribution - NonCommerciall 4.0 International 
Présence en Réunion de Concertation Pluridisciplinaire dans les cancers des Voies AéroDigestives Supérieures : vécu et satisfaction du patient.

D. Chaillou ${ }^{1}$, G. Mortuaire ${ }^{1}$, V. Deken-Delannoy ${ }^{2}$, B Rysman $^{1}$, D. Chevalier ${ }^{1}$, *F. Mouawad ${ }^{1 ; 3}$

1. Service d'ORL et de chirurgie cervico-faciale, hôpital Huriez, université de Lille, CHRU de Lille, rue Michel Polonovski, 59000 Lille, France.

2. Unité de Méthodologie - Biostatistique et Data Management, Maison Régionale de la recherche clinique, CHRU de Lille, rue du Professeur Laguesse, 59037 Lille, France.

3. Service d'ORL et de chirurgie cervico-faciale, INSERM U 908, hôpital Huriez, université de Lille, CHRU de Lille, rue Michel Polonovski, 59000 Lille, France.

*Auteur correspondant :

e-mail : francois.mouawad@chru-lille.fr (François MOUAWAD)

Tél. : +33320918950

\section{Déclaration de liens d'intérêt :}

Les auteurs déclarent ne pas avoir de liens d'intérêt. 


\section{Résumé}

Contexte : la Réunion de Concertation Pluridisciplinaire (RCP) permet une amélioration de la survie globale et une réduction du délai de prise en charge des patients atteints de cancers des VADS. Aucune étude ne s'est intéressée au ressenti des patients qui assistent à cette réunion. Nous voulions donc répondre à deux questions: cette réunion a-t-elle un impact anxiodépressif sur les patients présents et quelle est leur satisfaction au terme de celle-ci ?

Patients et Méthodes : étaient inclus, les patients présents en RCP ayant accepté de participer à l'étude et ayant complètement rempli l'ensemble des questionnaires. Le questionnaire HAD (évaluation de l'anxiété et de la dépression) et un questionnaire de satisfaction étaient complétés, à différents temps, par le patient : T0 avant la RCP, T1 après la RCP et T2 à 1 mois de la RCP pour le questionnaire $\mathrm{HAD}$ et à $\mathrm{T} 1$ et $\mathrm{T} 2$ pour le questionnaire de satisfaction.

Résultats: il n'existait pas de différence significative du nombre de patients anxieux en fonction des temps T0 et T1 ( $\mathrm{p}=0,6085)$, T0 et T2 $(\mathrm{p}=1)$ et $\mathrm{T} 1$ et $\mathrm{T} 2(\mathrm{p}=1)$. Il n'existait pas de différence significative du nombre de patients dépressifs en fonction des temps $\mathrm{T} 0$ et $\mathrm{T} 1$ $(\mathrm{p}=0,9397)$, $\mathrm{T} 0$ et $\mathrm{T} 2(\mathrm{p}=1)$ et $\mathrm{T} 1$ et $\mathrm{T} 2(\mathrm{p}=1)$. La satisfaction moyenne des patients, était élevée (Q14 : 8,7/10 à T1 et 7,7/10 à T2) mais était significativement modifiée avec un score qui diminuait entre $\mathrm{T} 1$ et $\mathrm{T} 2(\mathrm{p}=0,0009<0,05)$. Le pourcentage d'informations retenues $(\mathrm{Q} 12)$ par les patients était significativement diminué entre T1 $(86 \%$ des informations retenues en moyenne, écart-type de 0,2 et médiane de $94 \%)$ et T2 (78\% des informations retenues en moyenne, écart-type de 0,2 et médiane de $81 \%$ ) ( $\mathrm{p}=0,03$ ). La RCP en présence du patient ne semble pas créer ou majorer une anxiété ou un syndrome dépressif préexistant.

Mots-clés : Réunion de concertation Pluridisciplinaire, cancer des Voies Aéro-Digestives Supérieures, vécu patient, anxiété, dépression. 


\section{Introduction}

La réunion de concertation pluridisciplinaire $(\mathrm{RCP})$ est obligatoire depuis la circulaire du Ministère de la santé du 22/02/2005. Son avis reste toutefois consultatif.

Elle doit compter au moins 3 praticiens de spécialités différentes pour respecter le quorum. Les acteurs peuvent être : chirurgien ORL ou maxillo-facial, radiothérapeute, oncologue, radiologue, médecin nucléaire, anatomo-pathologiste, chirurgien reconstructeur [1]. L'ensemble des éléments constitutifs du dossier médical est présenté lors de cette réunion : l'histoire de la maladie, l'examen clinique, la description de l'examen endoscopique, le résultat anatomopathologique et l'imagerie. La proposition thérapeutique est établie en présence du patient ou sur dossier médical après discussion entre les différents intervenants. Le protocole proposé se fonde sur des référentiels de traitements régionaux, nationaux ou internationaux eux-mêmes basés sur les données scientifiques les plus récentes [2]. La proposition de protocole thérapeutique est adressée le lendemain de la RCP aux différents intervenants et correspondants médicaux actuels et futurs du patient afin de débuter le plus précocement possible le traitement. Le patient reste au cœur de la proposition thérapeutique et au projet de soins qui lui est proposé [3]. Bien qu'acteur de la décision thérapeutique, le malade assiste ou non à la RCP en fonction des pratiques des différents services.

Certains centres optent pour une consultation dédiée entre le patient et son chirurgien otorhino-laryngologiste (ORL) référent pour faire part de la prise en charge proposée en RCP. Dans notre service, les patients sont majoritairement présents à cette réunion, la consultation d'annonce du cancer est réalisée au préalable et permet d'expliquer la RCP. En effet, l'intéressé peut assister à cette réunion avec une personne de confiance et parfois d'autres accompagnants ou peut se présenter seul face à un certain nombre de praticiens qu'il ne connait pas mais dont la présence lui a été précisée. Ces éléments peuvent parfois être traumatisants ou générer un syndrome anxiodépressif. 
L'instauration de la RCP a permis de réduire de façon significative le délai de prise en charge entre le diagnostic anatomopathologique et le début du traitement [4] mais également d'améliorer le taux de survie [5]. Dans 10\% des cas une modification étaiot faite au protocole thérapeutique après un second avis en RCP et dans $49 \%$ des cas le diagnostic devait être complété ou précisé soit par relecture de l'imagerie soit par des analyses anatomopathologiques complémentaires [4]. Peu d'études en cancérologie tête et cou se sont intéressées à la satisfaction des patients présents, ou à l'état anxiodépressif après passage en RCP.

L'objectif principal de ce travail était donc d'évaluer par le biais de questionnaires adaptés l'impact psychologique avant, immédiatement après et à un mois après la RCP et la satisfaction des patients au décours et à un mois de cette réunion.

\section{Patients et méthodes}

L'étude menée est un travail mono centrique et prospectif réalisé dans notre service d'ORL et de chirurgie cervico-faciale entre le 1er Décembre 2016 et le 2 Mars 2017.

\section{Population}

\section{I.1 Critères d'inclusion :}

Les patients inclus répondaient à certains critères. Il s'agissait de patients présentant un premier cancer des voies aérodigestives supérieures (VADS) confirmé en histologie, avec une première présentation en RCP, ayant signé le consentement de participation à l'étude après lecture de la lettre d'information et ayant répondu en entier et à la totalité des questionnaires.

\section{2 Critères d'exclusion :}

Les patients de moins de 18 ans ou présentant un antécédent de cancer des VADS et/ou avec précédent passage en RCP pour un autre organe étaient exclus du travail. De même, les patients illettrés ou ne maîtrisant pas la langue française, ou présentant des troubles cognitifs sévères, sous tutelle, ne permettant pas la compréhension et la bonne participation à l'étude 
étaient exclus. Les patients qui n'avaient pas respecté les consignes de remplissage des questionnaires ou qui avaient incomplètement rempli ceux-ci étaient exclus.

\section{Schéma de l'étude}

Une présélection des patients incluables selon les critères précédemment cités, était réalisée avant la RCP, sur la liste de présence informatique, par le médecin menant le travail. Le patient était accueilli dans le service où une lettre d'information présentant l'étude et un consentement à signer lui était remis. Les patients acceptant l'étude complétaient, sans aucune aide médicale ou paramédicale, le questionnaire Hospital Anxiety and Depression (HAD) avant d'assister à la RCP. La totalité des patients inclus ont bénéficié d'un passage en RCP. Après la réunion, le patient complétait à nouveau sans aucune aide médicale ou paramédicale, l'échelle HAD ainsi que le questionnaire « ressenti de la RCP » avant de les rendre aux infirmières de consultation puis ceux-ci étaient remis au médecin réalisant l'étude. L'ensemble des réponses était ensuite rendu anonyme.

Le questionnaire HAD était donc remis au patient avant son passage en RCP (temps T0), puis juste après la RCP (temps T1) et envoyé à son domicile à 1 mois après la réunion (temps T2). Le questionnaire RCP était remis au patient juste au décours de la RCP (temps T1) et envoyé à son domicile à 1 mois (temps T2). Au temps T2, les questionnaires étaient envoyés au domicile des patients par la poste accompagnés d'une enveloppe pré-timbrée pour faciliter leur renvoi dans le service d'ORL après avoir été complétés par les patients.

Il était vérifié, dans le dossier médical, que l'évaluation à un mois se situait à au moins 10 jours avant le début de la ou des modalité(s) thérapeutique(s) décidées en RCP. Les réponses aux 2 questionnaires étaient colligées dans un tableur Excel. 


\section{Questionnaires}

\section{III.1 RCP (Annexe 1) :}

Nous avons élaboré un questionnaire centré sur la RCP. Il permet d'évaluer le ressenti et la satisfaction des patients après leur passage en RCP. Ce questionnaire a été soumis à la Délégation à la Recherche Clinique et à l'Innovation (DRCI) de notre structure hospitalière qui n'a pas émis d'objection à son utilisation.

Le questionnaire comprend 15 questions en totalité dont 12 questions qui attendent une réponse binaire simple par oui ou par non. Deux questions se présentent sous la forme d'une Echelle Visuelle Analogique (EVA) avec un score compris entre 0 et $10(0$ correspondant à $0 \mathrm{~cm}$ et 10 à $10 \mathrm{~cm})$. Le zéro correspond au jugement le plus négatif et 10 à la meilleure appréciation possible. Enfin, une seule question attend une réponse sous la forme d'un pourcentage, présenté à l'aide d'une règle, allant de 0 à $100 \%(0$ correspondant à $0 \mathrm{~cm}$ et $100 \%$ à $10 \mathrm{~cm})$. La possibilité d'écrire un commentaire libre est offerte à la fin du questionnaire. Ce questionnaire peut être rempli en quelques minutes (inférieur à 5 minutes).

\section{III.2 Questionnaire Hospital Anxiety Depression (HAD) (Annexe 2) :}

En 1983, l'étude de Zigmond et al [6] a permis de valider le questionnaire HAD concernant l'évaluation de l'anxiété et de la dépression chez les patients hospitalisés dans des structures non psychiatriques. Il s'agit d'un questionnaire court et dont les résultats ne sont pas influencés par l'aspect organique de la pathologie. Il ne prend en compte que la dimension psychique et il peut être répété. Il est très largement utilisé pour évaluer l'anxiété et la dépression chez les patients atteints de cancer et validé en cancérologie tête et cou [7]. Même s'il est bref, sa sensibilité et sa spécificité sont aussi robustes que celles du General Health Questionnaire (GHQ), du Beck Depression Inventory (BDI) ou du State-Trait Anxiety Inventory (STAI) [7]. Ce questionnaire se compose de 7 items évaluant la dépression avec des questions centrées principalement sur l'anhédonie secondaire à la dépression et de 7 items évaluant l'anxiété. Nous avons donc utilisé ce questionnaire dans l'évaluation de l'anxiété et 
la dépression de nos patients avant et après leur participation à la RCP. L'anxiété est évaluée par 7 questions cotées de 0 à 3 avec un score total allant de 0 à 21. La dépression est évaluée de la même façon. Un score global de 0 à 7 correspond à l'absence de symptômes anxieux ou dépressifs, un score de 8 à 10 ne permet pas de conclure et un score supérieur ou égal à 11 permet d'affirmer la symptomatologie anxieuse ou dépressive.

\section{Etude statistique}

L'analyse statistique a été réalisée au sein de l'unité de méthodologie bio statistique et data Management de notre structure hospitalière. Un recueil de données sous forme de tableau Excel a été envoyé. A chaque patient correspondait un numéro permettant un anonymat total. Les réponses au questionnaire RCP étaient colligées de la façon suivante :

- $\quad$ pour les items 1 à 8,10 à 11,13 et $15: 0$ si la réponse était NON et 1 si la réponse était OUI.

- $\quad$ pour les items 9, 12 et 14, il s'agissait respectivement d'un score sur 10, d'un pourcentage et d'un score sur $10(0$ correspondant à $0 \mathrm{~cm}$ et 10 ou $100 \%$ correspondant à $10 \mathrm{~cm})$.

- $\quad$ tous les items étaient comparés statistiquement entre T1 et T2.

Les réponses au questionnaire HAD étaient colligées de la façon suivante :

- $\quad 0$ en cas d'absence de symptômes anxieux ou dépressifs (score global d'anxiété ou de dépression compris entre 0 et 7)

- 1 en cas de symptomatologie anxieuse ou dépressive affirmée (score global d'anxiété ou de dépression supérieur ou égal à 11)

- 2 en cas de symptomatologie anxieuse ou dépressive douteuse (score global d'anxiété ou de dépression entre 8 et 10).

Pour le questionnaire HAD, il s'agissait de comparer les scores d'anxiété et de dépression aux différents temps T0, T1 et T2. Il s'agissait également de déterminer si certains patients classés 
en « syndrome anxieux », en «absence de syndrome anxieux » ou en «syndrome dépressif » et «absence de syndrome dépressif » changeaient de catégorie au cours du temps. Pour cela nous avons décidé de ne plus classer les patients qu'en 2 catégories : syndrome présent ou syndrome absent. Les patients classés « douteux anxieux » ou «douteux dépressif » ont donc été réunis avec les patients ne présentant pas de syndrome anxieux ou pas de syndrome dépressif respectivement.

Pour le questionnaire RCP, il s'agissait d'évaluer s'il existait une modification des réponses des patients en fonction des temps $\mathrm{T} 1$ et $\mathrm{T} 2$.

Les résultats sont exprimés avec la moyenne, écart-type, médiane et range pour les variables numériques et avec les effectifs et pourcentages pour les variables qualitatives. La normalité des distributions a été évaluée à l'aide d'histogrammes et du test de Shapiro-Wilk. L'analyse de l'évolution, des paramètres d'anxiété et de dépression au cours du temps ainsi que par rapport au stade du cancer (stades précoces 1 et 2 versus stades avancés 3 et 4 ), a été réalisée par une étude de la variance pour mesures répétées utilisant le modèle linéaire mixte avec une correction de Bonferroni pour les analyses en post-hoc. L'analyse comparative entre les questionnaires juste après la RCP et à 1 mois a été obtenue en utilisant le test de McNemar pour les variables binaires et par le test de Student apparié en cas de distribution normale ou le test de Wilcoxon apparié autrement.

Le niveau de significativité a été fixé à 5\%. Les analyses statistiques ont été effectuées à l'aide du logiciel SAS (SAS Institute version 9.3).

\section{Résultats}

\section{Epidémiologie}

Entre le $1^{\text {er }}$ Décembre 2016 et le 2 Mars 2017, 255 dossiers ont été discutés en RCP dont 144 patients présents et 74 patients éligibles. Sur ces 74 patients, 54 ont accepté de participer à notre étude mais seulement 34 patients $(63 \%)$ ont rempli correctement et précisément 
l'ensemble des questionnaires aux différents temps T0, T1 et T2. Notre population était constituée de 25 hommes (73,5\%) et 9 femmes (26,5\%), soit un sex-ratio de 2,78/1, âgés en moyenne de 60,5 ans avec une médiane de 60 ans [25 ans ; 73 ans]. Les lésions de la cavité buccale et de l'oropharynx représentaient respectivement 9 cas chacune, suivies par les lésions du larynx avec 8 cas puis du sinus piriforme avec 3 cas. Un cas de lésion de fosse nasale était présent et les adénopathies sans porte d'entrée représentaient 4 cas. Selon la classification TNM, deux lésions étaient classées Tis (in situ), 7 cas T1, 8 cas T2, 6 cas T3, 7 cas T4, 4 cas Tx. La moitié des patients (17 cas) présentaient un statut ganglionnaire N0, 7 patients étaient classés N1, 1 cas N2a, 2 patients $\mathrm{N} 2 \mathrm{~b}, 5$ cas $\mathrm{N} 2 \mathrm{c}$ et 1 cas $\mathrm{N} 3$, un patient était Nx. Seuls deux patients présentaient un statut métastatique M1 (Tableau 1).

\section{Résultats questionnaire HAD}

II.1 Analyse statistique descriptive :

Au temps T0, sur 34 patients, 12 ne présentaient aucun syndrome anxieux, 10 étaient anxieux et 12 étaient classés en douteux. Chez 22 patients aucun syndrome dépressif n'était mis en évidence, 6 présentaient une dépression et 6 étaient classés en douteux.

Au temps T1, 13 des patients ne présentaient aucun syndrome anxieux, 14 en présentaient un et 7 patients étaient classés en douteux. Chez 24 patients aucun syndrome dépressif n'était retrouvé, 3 étaient dépressifs et 7 étaient classés en douteux.

Au temps T2, 11 patients ne présentaient aucun syndrome anxieux, 12 étaient anxieux et 11 étaient classés douteux. Chez 20 patients aucun syndrome dépressif n'était retrouvé, 5 étaient classés dépressifs et 9 étaient douteux.

Il n'y avait pas de différence observée dans le nombre de patients anxieux $(p=0,51)$ ou dépressifs $(\mathrm{p}=0,97)$ en fonction du stade tumoral précoce (stades 1 et 2$)$ et avancé (stades 3 et 4). 
II.2 Analyse statistique comparative aux différents temps :

Le score total d'anxiété n'était pas significativement modifié au cours du temps. Il n'existait pas de différence significative entre le score total d'anxiété au temps T0 (médiane 10 [6-12]) et au temps T1 (médiane 10 [5-13]) avec un p=1. Il n'existait pas non plus de différence significative entre T0 et T2 $(\mathrm{p}=1)$ ni entre $\mathrm{T} 1$ et $\mathrm{T} 2(\mathrm{p}=1)$ (Figure 1).

En ce qui concerne la présence ou non d'un syndrome anxieux (syndrome anxieux si score d'anxiété $\geq 11$, absence de syndrome anxieux si score d'anxiété $\leq 10$ ), il n'existait pas non plus de différence significative entre les différents temps T0 et $\mathrm{T} 1(\mathrm{p}=0,6085)$, T0 et $\mathrm{T} 2(\mathrm{p}=1)$ et $\mathrm{T} 1$ et $\mathrm{T} 2(\mathrm{p}=1)$.

Il existait une différence significative entre le score total de dépression au temps T1 (médiane 5[3-8]) et au temps T2 (médiane 7 [4-9]) avec un p=0,04. Celui-ci est donc modifié 1 mois après la RCP avec un score qui augmente de façon significative. Par contre, il n'existe pas de différence significative du score total de dépression entre les temps T0 et T1 (p=1) ni entre les temps T0 et $\mathrm{T} 2(\mathrm{p}=0,22)$ (Figure 2$)$.

Dans l'analyse de la présence ou non d'un syndrome dépressif (syndrome dépressif si score de dépression $\geq 11$, absence de syndrome dépressif si score de dépression $\leq 10$ ), il n'existait pas de différence significative entre les différents temps T0 et T1 ( $\mathrm{p}=0,9397)$, T0 et T2 ( $\mathrm{p}=1)$ et $\mathrm{T} 1$ et $\mathrm{T} 2(\mathrm{p}=1)$.

Il n'y avait pas de différence observée dans le nombre de patients anxieux ( $p=0,88$ à T0, $\mathrm{p}=0,53$ à $\mathrm{T} 1$ et $\mathrm{p}=0,21$ à $\mathrm{T} 2)$ ou dépressifs $(\mathrm{p}=0,13$ à $\mathrm{T} 0, \mathrm{p}=0,14$ à $\mathrm{T} 1$ et $\mathrm{p}=0,20$ à $\mathrm{T} 2)$ en fonction du stade tumoral précoce (stades 1 et 2) et avancé (stades 3 et 4) aux différents temps T0, T1 et T2.

\section{Résultats questionnaire RCP}

III.1 Analyse statistique descriptive (Tableau 2) :

La grande majorité des patients n'ont pas trouvé le temps d'attente trop long avant leur passage en $\mathrm{RCP}(\mathrm{Q} 1$ : 88,2\% à $\mathrm{T} 1$ et $91,2 \%$ à $\mathrm{T} 2)$ et n'ont pas été gênés par la présence de 
patients déjà traités en salle d'attente (100\% de réponses négatives à Q2). Ils ont considéré l'attente du jour de la RCP majoritairement stressante à T1 mais moins, à posteriori, à T2 (Q3 : 52,9\% à T1 et 47,1\% à T2). Les patients ont considéré en grande partie la salle de RCP appropriée à $88,2 \%$ à $\mathrm{T} 1$ versus $91,2 \%$ à T2 (Q6). Pour 76,5\% des malades à T1 et 81,8\% à T2, les spécialistes présents se sont présentés aux soignés (Q7). La gêne moyenne ressentie par le patient devant la présence d'autant de praticiens était évaluée à 3,8/10 à T1 contre 5,1/10 à T2 (Q8). Par ailleurs, le patient ne semblait quasiment jamais gêné par un examen clinique devant tous ces praticiens (Q9 : 3\% à T1 et 6,1\% à T2). La totalité des patients a compris les informations sur sa pathologie et le vocabulaire employé à T1 mais un peu moins à T2 (Q10 et Q11) et un large pourcentage d'informations semble avoir été compris par les patients (Q12: 86\% des informations à T1 et 78\% à T2). Une large part des patients a pu poser des questions lors de la RCP (Q13 : 90,9\% à T1 et 87,9\% à T2).

La satisfaction moyenne des patients, au sujet de la RCP, était élevée (Q14 : 8,7/10 à T1 et 7,7/10 à T2). Une grande majorité de patients étaient informés du déroulement de la RCP et des différents traitements possibles (Q4 et Q5 : respectivement 73,5\% et 85,3\% à T1).

III.2 Analyse statistique comparative aux différents temps :

L'analyse statistique n'a pas mis en évidence de différence significative entre T1 et T2 pour les questions suivantes: Q1 ( $\mathrm{p}=0,32), \mathrm{Q} 3(\mathrm{p}=0,41), \mathrm{Q} 4(\mathrm{p}=1), \mathrm{Q} 5(\mathrm{p}=0,34), \mathrm{Q} 6(\mathrm{p}=0,65), \mathrm{Q} 7$ $(\mathrm{p}=1)$, Q9 (p=0,56), Q13 ( $\mathrm{p=0,56).} \mathrm{Il} \mathrm{n’y} \mathrm{avait} \mathrm{donc} \mathrm{pas} \mathrm{de} \mathrm{différence} \mathrm{significative} \mathrm{observée}$ entre le moment de la RCP et à un mois, dans l'interprétation faite par le patient, à posteriori, qui restait favorable :

- Pour l'attente du jour de la RCP,

- L'attente dans la salle d'attente avant d'entrée dans le bureau,

- Sur les informations délivrées pour la RCP et les possibilités thérapeutiques,

- Sur la possibilité de poser l'ensemble de ses questions pendant la réunion, 
- Sur l'adaptation de la salle de RCP à l'accueil du patient,

- Sur la présentation des médecins lors de la réunion et sur la gêne liée à l'examen clinique lors de la réunion.

Aucun test de comparaison n'a pu être réalisé pour les questions 2, 10, 11 et 15 en raison d'un effectif insuffisant.

Pour la question 8 relative à la gêne ressentie par les patients face au nombre de spécialistes, il n'existe pas de différence significative entre $\mathrm{T} 1$ et $\mathrm{T} 2(\mathrm{p}=0,06)$. La gêne moyenne ressentie par le patient est évaluée à 3,81/10 (avec un écart-type de 3,8 et une médiane de 3,6) à T1 et 5,1/10 (écart-type de 3,7 et médiane de 5,4) à T2. Dans la question concernant le pourcentage d'informations retenues (Q12) par les patients, nous avons trouvé une diminution significative entre T1 (86\% des informations retenues en moyenne, écart-type de 0,2 et médiane de 94\%) et T2 (78\% des informations retenues en moyenne, écart-type de 0,2 et médiane de $81 \%$ ) $(\mathrm{p}=0,03)$. Enfin, la satisfaction globale est significativement modifiée avec un score qui diminue entre T1 et T2 (p=0,0009<0,05). En effet, à T1, la satisfaction globale est de 8,7/10 en moyenne (écart-type de 1,8 et une médiane de 9,3) et de 7,7/10 à T2 (écart-type de 2,3 et médiane de 8,2) (Q14).

\section{Discussion}

Tous les dossiers des patients présentant un cancer des VADS sont discutés en RCP. Cette dernière a été rendue obligatoire depuis 2005 par le Ministère de la Santé qui bien qu'ayant établi certaines règles, dont la nécessité d'un quorum de praticiens nécessaires à son bon déroulement, n’a pas donné de précision sur la présence ou non du patient à cette réunion. Dans notre service, historiquement, les comités de décision cancérologique qui sont devenus les RCP sont réalisés, en majorité, en présence du patient. En effet, lorsqu'il s'agit d'une première tumeur, que l'état général est nécessaire à l'évaluation thérapeutique et que le patient souhaite assister à la réunion, cette modalité lui est proposée mais jamais imposée. 
Afin d'obtenir une interprétation rigoureuse des questionnaires, nous souhaitions que ceux-ci soient parfaitement remplis sans aucune aide extérieure médicale ou paramédicale (complètement et en respectant les consignes). Néanmoins sur les 54 patients incluables, 20 ont étaient exclus car des questionnaires étaient incomplets (une ou plusieurs réponses manquantes) ou présentaient des commentaires pouvant impacter l'interprétation.

L'impact de la RCP sur le taux de survie est établi. En effet en 2011, Friedland et al. ont comparé le taux de survie à 5 ans chez des patients présentant un cancer des VADS stade IV et dont la prise en charge thérapeutique a été actée en RCP, au taux de survie à 5 ans chez des patients présentant le même stade tumoral mais sans décision thérapeutique collégiale. Cette étude a montré que les premiers avaient un meilleur taux de survie à 5 ans et ce de manière significative [8]. Une autre série, menée par Wang en 2012, a mis en évidence une réduction du risque relatif de décès chez des patients atteints d'un cancer de la cavité buccale traités, après discussion en RCP versus ceux n'ayant pas bénéficié d'une décision collégiale [9]. En 2007, Butow et al. se sont intéressés à la RCP dans le cadre du cancer du sein. Ils ont montré que $93 \%$ des représentantes (uniquement des femmes atteintes elles-mêmes de cancer du sein) d'associations de patientes atteintes de cancer du sein étaient favorables à la présence des patientes en RCP [10]. En revanche, moins d'un tiers des chirurgiens et des oncologues y étaient favorables. Néanmoins, aucune étude ne s'est intéressée à cette présence ou non du patient en RCP, à son ressenti, dans la pathologie cancéreuse des VADS.

\section{Satisfaction RCP}

Stalfors et al. ont étudié la satisfaction des patients lors de leur participation à la RCP VADS [11]. Les patients étaient divisés en 2 groupes : des patients présents physiquement à la RCP avec leur ORL référent et des patients assistant à la RCP, via un système de télémédecine, mais avec leur médecin traitant à leur côté. Les réponses aux questionnaires de satisfaction différaient peu en dehors des réponses aux deux questions suivantes : «J'avais l'impression 
que tout le monde parlait de moi mais sans s'adresser directement à moi » et « Je me sentais rassuré d'avoir mon médecin à mes côtés » (médecin traitant pour le groupe télémédecine ou médecin ORL de l'hôpital local pour les patients présents). A la première question, les patients présents en RCP y ont plus majoritairement répondu « oui » que dans le groupe télémédecine avec une différence significative. A la deuxième question, le groupe télémédecine y a répondu plus souvent «oui » et ce de façon significative par rapport au groupe de patients présents. Ces éléments apparaissent intéressants puisqu'ils montrent que lorsque les patients sont présents, ils souhaitent être au centre de la décision et pouvoir exprimer leur point de vue et leurs doutes à travers des interrogations posées au corps médical. Lorsque ce contact direct ne peut être établi et que la réunion se déroule sous forme d'une visioconférence, les patients souhaitent être soutenus par une personne de confiance, dans cette étude, leur médecin traitant. Dans notre travail, il n'y a pas d'évaluation de la télémédecine (non utilisée) mais nous observons, lorsque les patients sont présents avec leur ORL référent, une note de satisfaction globale élevée avec près de $8 / 10$ patients satisfaits au moment de la réunion mais également à posteriori. Dans l'étude de Choy et al. [12], 91\% des patients déclaraient avoir mieux compris leur maladie, $71 \%$ les traitements proposés et $84 \%$ se disaient mieux informés. Ces données sont comparables à celles de notre série avec plus de $90 \%$ des patients ayant compris les informations données en RCP et $86 \%$ des informations retenues à T1 et $78 \%$ à T2, même si à posteriori le taux de compréhension diminue en rapport probablement avec l'intervalle par rapport à la réunion. De plus, il n'apparait pas que les patients aient été gênés par l'examen clinique ou bridés dans leurs interrogations.

L'attente avant le passage en RCP ne semble pas particulièrement désagréable aux patients de même que la présence de malades traités ou l'inconfort de la salle d'examen. Ces éléments auraient pu paraitre désagréables pour des observateurs extérieurs (personnel médical et paramédical). Néanmoins, le nombre de praticiens présents dans la salle de RCP semble à 
posteriori apporter un dérangement non négligeable aux malades. La possibilité d'une présence du patient dans une salle d'examen dédiée avec le quorum d'examinateurs et une visioconférence permettant la retransmission, dans une autre salle avec présence d'autres praticiens seniors ou en formation, de l'examen et de la discussion, permettrait de diminuer cette gêne exprimée par les patients.

\section{Score HAD}

Nous n'avons pas mis en évidence d'augmentation de l'état d'anxiété des patients immédiatement et un mois après la RCP ce qui rejoint l'étude de Choy et al. [12]. En effet, dans cette étude il n'y avait pas de différence significative en termes d'anxiété chez les patients atteints de cancer du sein et participant à la RCP et ceux n'y étant pas. Le questionnaire d'anxiété utilisé dans la série de Choy et al. était le STAI. Dans notre étude, le questionnaire utilisé est le HAD reconnu et validé en cancérologie tête et cou pour évaluer le degré d'anxiété et de dépression [13]. Toutefois, la participation à la RCP pourrait davantage être considérée comme un stress aigu subi par le patient pour lequel le questionnaire HAD n'est peut-être pas le plus adapté. Il n'existe pas à ce jour de questionnaire dédié à cette situation et validé en cancérologie des VADS.

Par ailleurs, notre étude a mis en évidence une augmentation significative du score total de dépression entre $\mathrm{T} 1$ (juste après la $\mathrm{RCP}$ ) et $\mathrm{T} 2$ (un mois après la $\mathrm{RCP}$ ). Durant cet intervalle, les patients ont bénéficié de soins de support et ont pris de ce fait conscience de leur maladie cancéreuse pouvant constituer un biais dans l'interprétation de cette augmentation. En revanche, il n'existe pas de différence significative du nombre de patients présentant ou non un syndrome anxieux ou un syndrome dépressif au cours du temps. Il n'apparait donc pas que l'état d'anxiété ou le syndrome dépressif soient modifiés par la participation à la RCP. Néanmoins, il pourrait être intéressant de cibler cette partie de la population (entre T1 et T2) 
pour lui proposer plus systématiquement une prise en charge onco psychologique avant de débuter le protocole thérapeutique.

Le coût de la RCP n'a pas été analysé lors de ce travail. Dans le cadre des dépenses de santé, il serait intéressant d'évaluer le coût global de cette réunion (versant médecin et versant patient) et de le comparer avec la satisfaction globale, le vécu et la compliance au traitement du patient assistant à cette réunion versus un patient n'assistant pas à la RCP [14-15].

\section{Conclusion}

Le caractère obligatoire de la RCP est récent, son intérêt pour le malade et son bénéfice sur la survie est démontré dans la littérature. Néanmoins, peu d'études se sont intéressées au ressenti du soigné en cancérologie des VADS. La satisfaction apportée par la RCP en présence du patient, n'a été que peu explorée. Par ce travail, il est démontré que la RCP, en présence du patient n'est pas significativement inductrice d'anxiété et de syndrome dépressif. La satisfaction des patients est très positive. La présence d'une pluralité de domaines de compétences ne semble pas mal vécue et permet même au malade de questionner le corps médical et d'obtenir des informations claires tout en restant au centre de la décision médicale. 


\section{Références}

1. Circulaire DHOS/SDO/2005/10102/2008/99 relative à l'organisation des soins en cancérologie. Direction de l'Hospitalisation et de l'Organisation des soins. 22 Février 2005.

2. Shah BA, Qureshi MM, Jalisi S, et al. Analysis of decision making at a multidisciplinary head and neck tumor board incorporating evidence-based National Cancer Comprehensive Network (NCCN) guidelines. Pract Radiat Oncol. 2016;6(4):248-54.

3. Kelly SL, Jackson JE, Hickey BE, et al. Multidisciplinary clinic care improves adherence to best practice in head and neck cancer. Am J Otolaryngol. 2013;34(1):57-60.

4. Bergamini C, Locati L, Bossi P, et al. Does a multidisciplinary team approach in a tertiary referral centre impact on the initial management of head and neck cancer ? Oral Oncol. $2016 ; 54: 54-7$.

5. Licitra L, Keilholz U, Tahara M, et al. Evaluation of the benefit and use of multidisciplinary teams in the treatment of head and neck cancer. Oral Oncol. 2016 ;59:73-9.

6. Snaith RP, Zigmond AS. The hospital anxiety and depression scale. Br Med J Clin Res Ed. 1 i 1986 ;292(6516):344.

7. Bjelland I, Dahl AA, Haug TT, et al. The validity of the Hospital Anxiety and Depression Scale. An updated literature review. J Psychosom Res. 2002;52(2):69-77.

8. Friedland PL, Bozic B, Dewar J, et al. Impact of multidisciplinary team management in head and neck cancer patients. Br J Cancer. 2011;104(8):1246-8.

9. Wang Y-H, Kung P-T, Tsai W-C, et al. Effects of multidisciplinary care on the survival of patients with oral cavity cancer in Taiwan. Oral Oncol. 2012;48(9):803-10.

10. Butow P, Harrison JD, Choy ET, et al. Health professional and consumer views on involving breast cancer patients in the multidisciplinary discussion of their disease and treatment plan. Cancer. 2007;110(9):1937-44.

11. Stalfors J, Holm-Sjögren L, Schwieler A, et al. Satisfaction with telemedicine presentation at a multidisciplinary tumour meeting among patients with head and neck cancer. J Telemed Telecare. 2003;9(3):150-5.

12. Choy ET, Chiu A, Butow P, et al. A pilot study to evaluate the impact of involving breast cancer patients in the multidisciplinary discussion of their disease and treatment plan. Breast Edinb Scotl. 2007;16(2):178-89.

13. Herrmann C. International experiences with the Hospital Anxiety and Depression Scale--a review of validation data and clinical results. J Psychosom Res. 1997;42(1):17-41.

14. Wheless SA, McKinney KA, Zanation AM. A prospective study of the clinical impact of a multidisciplinary head and neck tumor board. Otolaryngol--Head Neck Surg Off J Am Acad Otolaryngol-Head Neck Surg. 2010;143(5):650-4.

15. Ke KM, Blazeby JM, Strong S, et al. Are multidisciplinary teams in secondary care cost-effective? A systematic review of the literature. Cost Eff Resour Alloc CE. 2013;11(1):7. 
Figure 1 : Comparaison du score d'anxiété aux différents temps T0, T1 et T2. NS = Non Significatif.

Figure 2 : Comparaison du score de dépression aux différents temps T0, T1 et T2. S = Significatif. 
Tableau ${ }^{\circ} 1$ : statut épidemiologique des patients de la cohorte.

\begin{tabular}{|c|c|}
\hline & $\mathrm{N}=34(\%)$ \\
\hline Age & $60,5[25 ; 73]$ \\
\hline Sexe & $\begin{array}{l}\text { Hommes } 25(73,5 \%) \\
\text { Femmes } 9(26,5 \%)\end{array}$ \\
\hline Sex ratio & $2,78 / 1$ \\
\hline Site tumoral & $\begin{array}{l}\text { Oropharynx }=9(26,5) \\
\text { Cavité buccale }=9(26,5) \\
\text { Larynx = } 8(23,5) \\
\text { Sinus piriforme }=3(8,8) \\
\text { Fosse nasale = } 1(2,9) \\
\text { ASPE = } 4(11,8)\end{array}$ \\
\hline $\mathrm{T}$ & $\begin{array}{l}\text { Tis }=2(5,8) \\
\mathrm{T} 1=7(20,6) \\
\mathrm{T} 2=8(23,5) \\
\mathrm{T} 3=6(17,6) \\
\mathrm{T} 4=7(20,6) \\
\mathrm{Tx}=4(11,9)\end{array}$ \\
\hline $\mathrm{N}$ & $\begin{array}{l}\mathrm{N} 0=17(50) \\
\mathrm{N} 1=7(20,6) \\
\mathrm{N} 2 \mathrm{a}=1(2,9) \\
\mathrm{N} 2 \mathrm{~b}=2(5,9) \\
\mathrm{N} 2 \mathrm{c}=5(14,7) \\
\mathrm{N} 3=1(2,9) \\
\mathrm{Nx}=1(2,9)\end{array}$ \\
\hline $\mathrm{M}$ & $2(5,8)$ \\
\hline
\end{tabular}

ASPE: Adénopathie Sans Porte d'Entrée. 
Tableau $n^{\circ}$ 2: questionnaire ressenti de la RCP par le patient à $T 1$ (après $R C P$ ) et T2 (à un mois de la RCP) exprimé en pourcentage ou en moyenne ou médiane selon les questions, avec p (degré de significativité). NS : Non Significatif. FE : Faible Effectif. NC: Non Concerné.

\begin{tabular}{|c|c|c|c|c|c|}
\hline \multirow[t]{2}{*}{ Questions } & \multicolumn{2}{|c|}{ Oui $(\%)$} & \multicolumn{2}{|c|}{ Non (\%) } & \multirow[t]{2}{*}{$\mathbf{P}$} \\
\hline & $T 1$ & $T 2$ & $T 1$ & $T 2$ & \\
\hline $\begin{array}{l}\text { Q1/L'attente avant de rentrer dans la salle } \\
\text { de RCP vous a-t-elle paru longue? }\end{array}$ & 11,8 & 8,8 & 88,2 & 91,2 & $\begin{array}{l}\text { NS } \\
(p=0,32)\end{array}$ \\
\hline $\begin{array}{l}\text { Q2/ Avez-vous été perturbé par la présence } \\
\text { en salle d'attente de patients ayant déjà } \\
\text { bénéficié d'un traitement? }\end{array}$ & 0 & 2,9 & 100 & 97,1 & $\mathrm{FE}$ \\
\hline $\begin{array}{l}\text { Q3/ L'attente du jour de la RCP est-il } \\
\text { stressant? }\end{array}$ & 52,9 & 47,1 & 47,1 & 52,9 & $\begin{array}{l}\text { NS } \\
(p=0,41)\end{array}$ \\
\hline $\begin{array}{l}\text { Q4/ Aviez-vous été informé du déroulement } \\
\text { de la RCP? }\end{array}$ & 73,5 & 70,66 & 26,5 & 29,4 & $\begin{array}{l}\text { NS } \\
(p=1)\end{array}$ \\
\hline $\begin{array}{l}\text { Q5/ Aviez-vous été informé des différentes } \\
\text { possibilités de traitement? }\end{array}$ & 85,3 & 73,5 & 14,7 & 26,5 & $\begin{array}{l}\text { NS } \\
(p=0,34)\end{array}$ \\
\hline $\begin{array}{l}\text { Q6/ Avez-vous trouvé la salle de } \\
\text { consultation appropriée (confort, bruit...)? } \\
\text { Si non, pourquoi? }\end{array}$ & 88,2 & 91,2 & 11,8 & 8,8 & $\begin{array}{l}\text { NS } \\
(p=0,65)\end{array}$ \\
\hline $\begin{array}{l}\text { Q7/ Les principaux représentants de } \\
\text { l'équipe médicale se sont-ils présentés? }\end{array}$ & 76,5 & 81,8 & 23,5 & 18,2 & $\begin{array}{l}\text { NS } \\
(p=1)\end{array}$ \\
\hline $\begin{array}{l}\text { Q8/ Avez-vous été impressionné ou gêné par } \\
\text { le nombre de personnes en RCP? }\end{array}$ & \multicolumn{2}{|c|}{$\begin{array}{l}3,8 / 5,1 \\
(\mathrm{p}=0,06)\end{array}$} & \multicolumn{2}{|c|}{$3,6 / 5,4$} & \\
\hline $\begin{array}{l}\text { Q9/ L'examen clinique devant tous ces } \\
\text { représentants du corps médical vous a-t-il } \\
\text { gêné? }\end{array}$ & 3 & 6,1 & 97 & 93,9 & $\begin{array}{l}\text { NS } \\
(p=0,56)\end{array}$ \\
\hline $\begin{array}{l}\text { Q10/ Les informations qui vous ont été } \\
\text { apportées ont-elles été toutes comprises? }\end{array}$ & 100 & 90,9 & 0 & 9,1 & $\mathrm{FE}$ \\
\hline $\begin{array}{l}\text { Q11/ Le vocabulaire employé par les } \\
\text { médecins était-il compréhensible? }\end{array}$ & 100 & 91,2 & 0 & 8,8 & $\mathrm{FE}$ \\
\hline $\begin{array}{l}\text { Q12/ Quel pourcentage des informations } \\
\text { avez-vous retenu? }\end{array}$ & $\% \mathrm{~T} 1$ & $\mathrm{~T} 2$ & $5 / 78$ & & $\mathrm{p}=0,03$ \\
\hline Q13/Avez-vous pu poser vos questions? & 90,9 & 87,9 & 9,1 & 12,1 & $\begin{array}{l}\text { NS } \\
(p=0,56)\end{array}$ \\
\hline Q14/ Quel est votre satisfaction globale & \multicolumn{5}{|c|}{ Moyenne T1/T2 Médiane T1/T2 } \\
\hline
\end{tabular}




\begin{tabular}{|l|l|l|l|l|l|}
\hline concernant la $R C P$ ? & $8,7 / 7,7$ & \multicolumn{3}{|c|}{$9,3 / 8,3$} & $\mathrm{p}=0,0009$ \\
\hline $\begin{array}{l}\text { Q15/ Etait-ce difficile en termes } \\
\text { d'organisation de vous rendre à cette } \\
\text { réunion? }\end{array}$ & 8,8 & 6,1 & 91,2 & 93,9 & $\mathrm{FE}$ \\
\hline
\end{tabular}




\section{Annexe 1 :}

\section{Questionnaire évaluant le ressenti et la satisfaction des patients lors de la RCP}

3 premières lettres de votre Nom :

3 premières lettres de votre Prénom :

Ce questionnaire a pour but d'évaluer votre satisfaction ainsi que votre ressenti concernant la Réunion de Concertation Pluridisciplinaire à laquelle vous venez d'assister. Les questions 1 à $8 ; 10 ; 11 ; 13$ et 15 nécessitent une réponse par oui ou par non et les questions $9 ; 12$ et 14 sont des questions avec une évaluation par une échelle numérique, reportez-vous pour ces dernières questions à la deuxième page.

\begin{tabular}{|l|l|l|}
\hline & OUI & NON \\
\hline $\begin{array}{l}\text { Q.1. L'attente avant de rentrer dans la salle de RCP vous a-t-elle paru } \\
\text { longue? }\end{array}$ & & \\
\hline $\begin{array}{l}\text { Q.2. Avez-vous été perturbé par la présence en salle d'attente de } \\
\text { patients ayant déjà bénéficié d'un traitement? }\end{array}$ & & \\
\hline Q.3. L'attente du jour de la RCP est-il stressant? & & \\
\hline Q.4. Aviez-vous été informé du déroulement de la RCP? & \\
\hline $\begin{array}{l}\text { Q.5. Aviez-vous été informé des différentes possibilités de } \\
\text { traitement? }\end{array}$ & \\
\hline $\begin{array}{l}\text { Q.6. Avez-vous trouvé la salle de consultation appropriée (confort, } \\
\text { bruit...)? }\end{array}$ & & \\
\hline $\begin{array}{l}\text { Q.7. Les principaux représentants de l'équipe médicale se sont-ils } \\
\text { présentés? }\end{array}$ & & \\
\hline $\begin{array}{l}\text { Q.8. Avez-vous été impressionné ou gêné par le nombre de } \\
\text { personnes en RCP? }\end{array}$ & & \\
\hline $\begin{array}{l}\text { Q.9. L'examen clinique devant tous ces représentants du corps } \\
\text { médical vous a-t-il gêné ? }\end{array}$ & & \\
\hline $\begin{array}{l}\text { Q.10. Les informations qui vous ont été apportées ont-elles été toutes } \\
\text { comprises? }\end{array}$ & & \\
\hline $\begin{array}{l}\text { Q.11. Le vocabulaire employé par les médecins était-il } \\
\text { compréhensible? }\end{array}$ & & \\
\hline Q.12. Quel pourcentage des informations avez-vous retenu? & & \\
\hline Q.13. Avez-vous pu poser vos questions? & & \\
\hline Q.14. Quel est votre satisfaction globale concernant la RCP? & \\
\hline $\begin{array}{l}\text { Q.15. Etait-ce difficile en termes d'organisation de vous rendre à } \\
\text { cette réunion? }\end{array}$ & & \\
\hline
\end{tabular}


Q.9. L'examen clinique devant tous ces représentants du corps médical vous a-t-il

gêné ?

0

Mode d'emploi : Pour répondre, il vous suffit de faire une croix sur la flèche au niveau où se situe votre gêne.

Q.12. Quel pourcentage des informations avez-vous retenu?

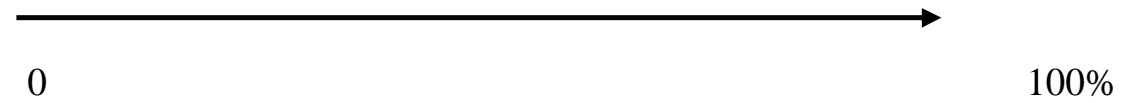

Mode d'emploi : Pour répondre, il vous suffit de faire une croix sur la flèche au niveau où se situe le pourcentage d'informations retenues.

Q.14. Quel est votre satisfaction globale concernant la RCP ?

0

10

Mode d'emploi : Pour répondre, il vous suffit de faire une croix sur la flèche au niveau où se situe votre niveau de satisfaction.

Merci pour vos réponses et le temps que vous avez consacré à remplir ce questionnaire. Nous vous remercions de bien vouloir remettre ce questionnaire rempli à une infirmière avant votre sortie. 


\title{
Annexe 2 : Questionnaire Hospital Anxiety Depression (HAD)
}

\author{
ECHELLE HAD \\ (Hospital ANXIETY AND Depression scale)
}

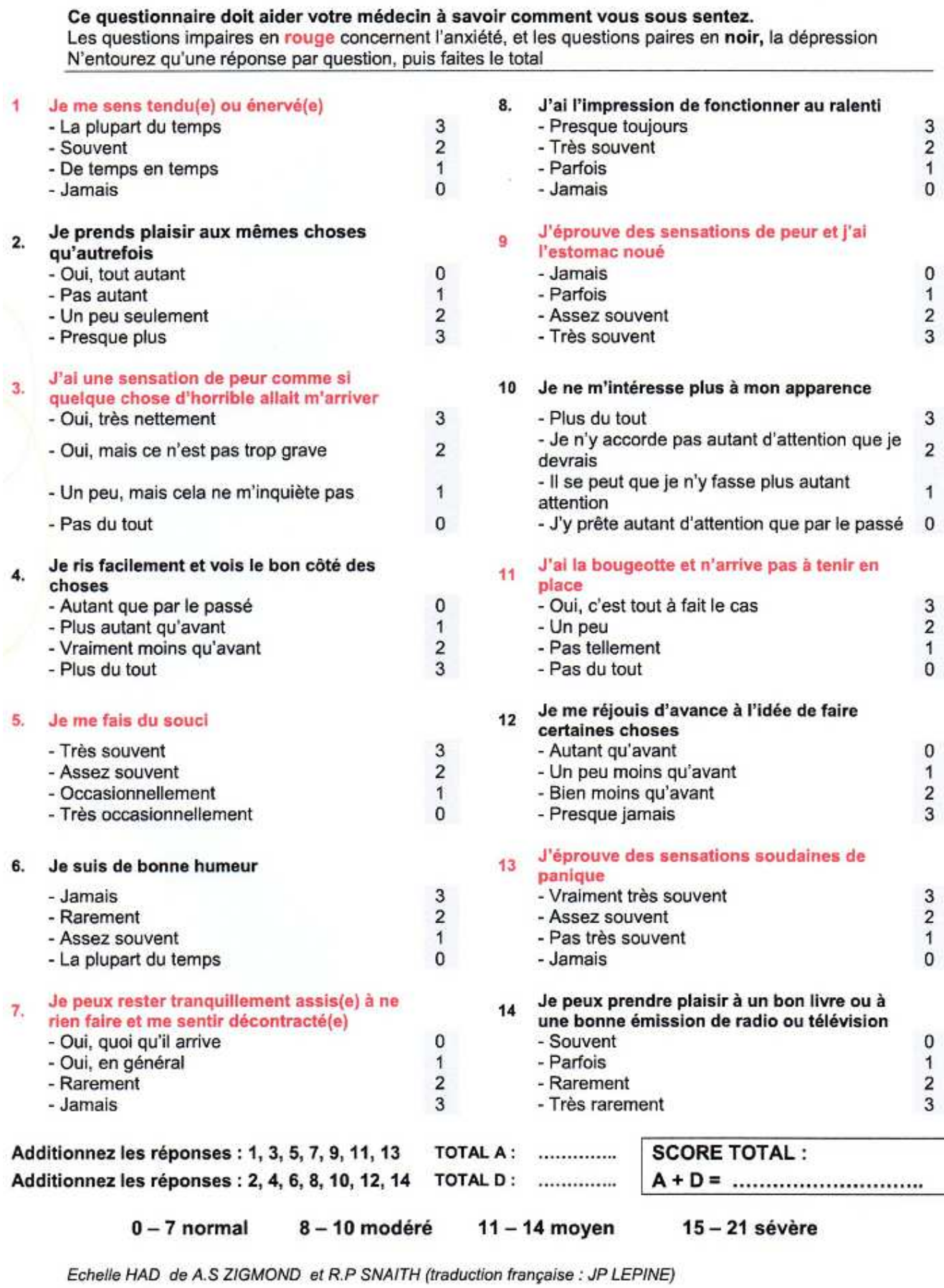

Echelle HAD de A.S ZIGMOND et R.P SNAITH (traduction française: JP LEPINE) 


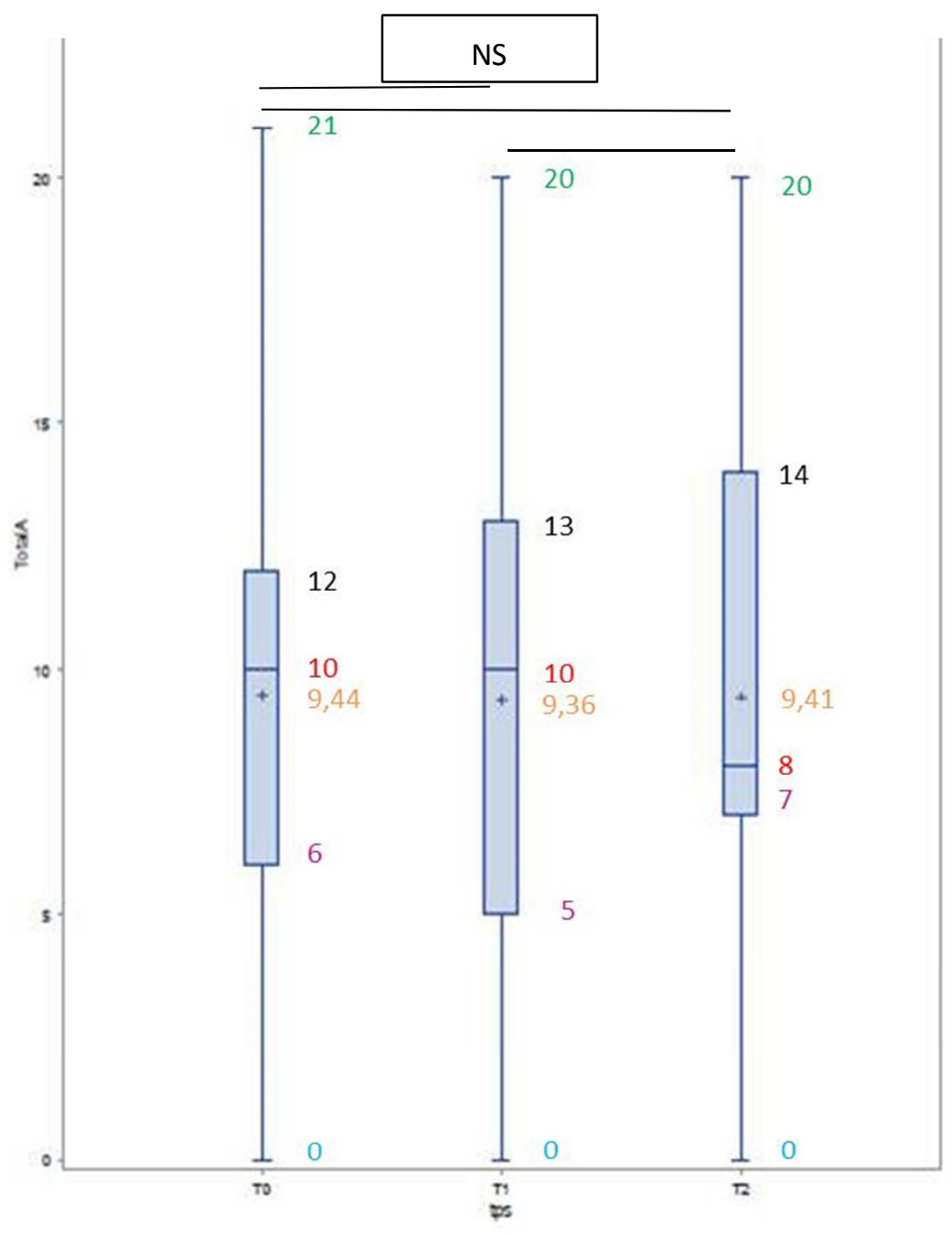

Maximum

Minimum

Moyenne

Médiane

$1^{\text {er }}$ quartile

$3^{\text {ème }}$ quartile 


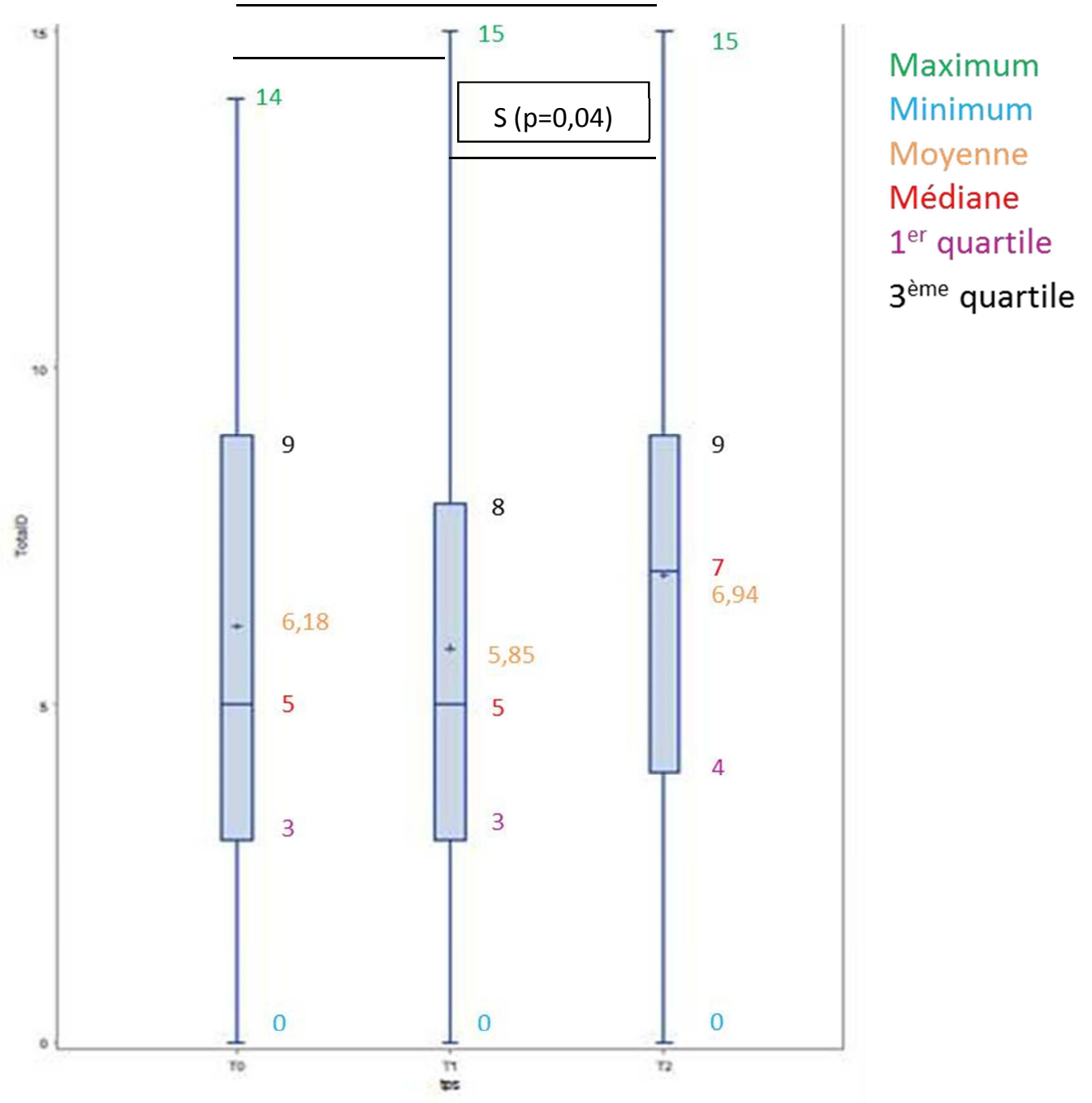

